\title{
Médecine de campagne et qualité de vie
}

François Burnier

Correspondance: Dr François Burnier Médecine générale FMH 8 , rue de l'Auberge CH-1142 Pampigny

francois.burnier@gmx.ch
Perdu dans la nature, loin de tout, toujours de garde, sollicité à toute heure par des malades pas forcément malades qui viennent sonner directement à sa porte...

L'image du médecin de campagne isolé, harassé, au bord de la déprime, est encore bien présente dans la population, et même singulièrement parmi ses confrères citadins. Il est vrai que, notamment dans les régions de montagne, certains médecins travaillent dans des conditions particulièrement difficiles. Cela ne constitue toutefois pas la règle générale, ainsi que je vais chercher à le montrer en évitant toute généralisation, me limitant à décrire ma situation personnelle.

J'ai décidé d'être généraliste en même temps que j'ai choisi d'étudier la médecine. C'était en 1963. Je crois que j'étais le seul de la volée à avoir d'emblée fait ce choix. «Tu es fou!» disaient les uns. «Tu n'as donc pas d'ambition?» demandaient des autres (si: j'ai l'ambition de faire de la bonne médecine générale, et de la pratiquer à la campagne. D'ailleurs, mon père était lui-même un généraliste heureux).

$\mathrm{Au}$ cours de mon année de chirurgie à l'Hôpital d'Aigle, j'ai rencontré une collègue bâloise, assistante en chirurgie à Sion, qui se destinait elle aussi à la médecine générale. Nous nous sommes mariés en
1973 et nous avons fait une moitié de nos années d'assistanat en Suisse romande et l'autre à Bâle, ville où est née notre fille, qui elle aussi est devenue médecin. Deux ans plus tard, notre fils naissait à la maternité de Saint-Loup, dans ce qui était alors l'hôpital de district.

\section{Un endroit idéal}

Sur le plan professionnel, la campagne est l'endroit par excellence où le généraliste est à sa place et où il peut utiliser les connaissances acquises non seulement en médecine interne mais aussi en petite chirurgie, gynécologie, pédiatrie, ORL, dermatologie et autres spécialités. C'est tout naturellement qu'il est consulté en premier, ce qui lui donne l'occasion de voir les patients «à l'état brut». Je n'oublierai jamais cette visite à domicile motivée, m'avait-on dit, par une grippe. Quoi de plus banal? Or les symptômes du patient m'ont mis sur la piste d'un botulisme, bel et bien confirmé par la suite.

J'aime la variété des patients et des problèmes qu'il faut résoudre. Cela devient particulièrement intéressant lorsqu'on cherche à aborder la personne dans son entité et dans son contexte social. Soucis de famille, problèmes de travail, dépendances diverses,

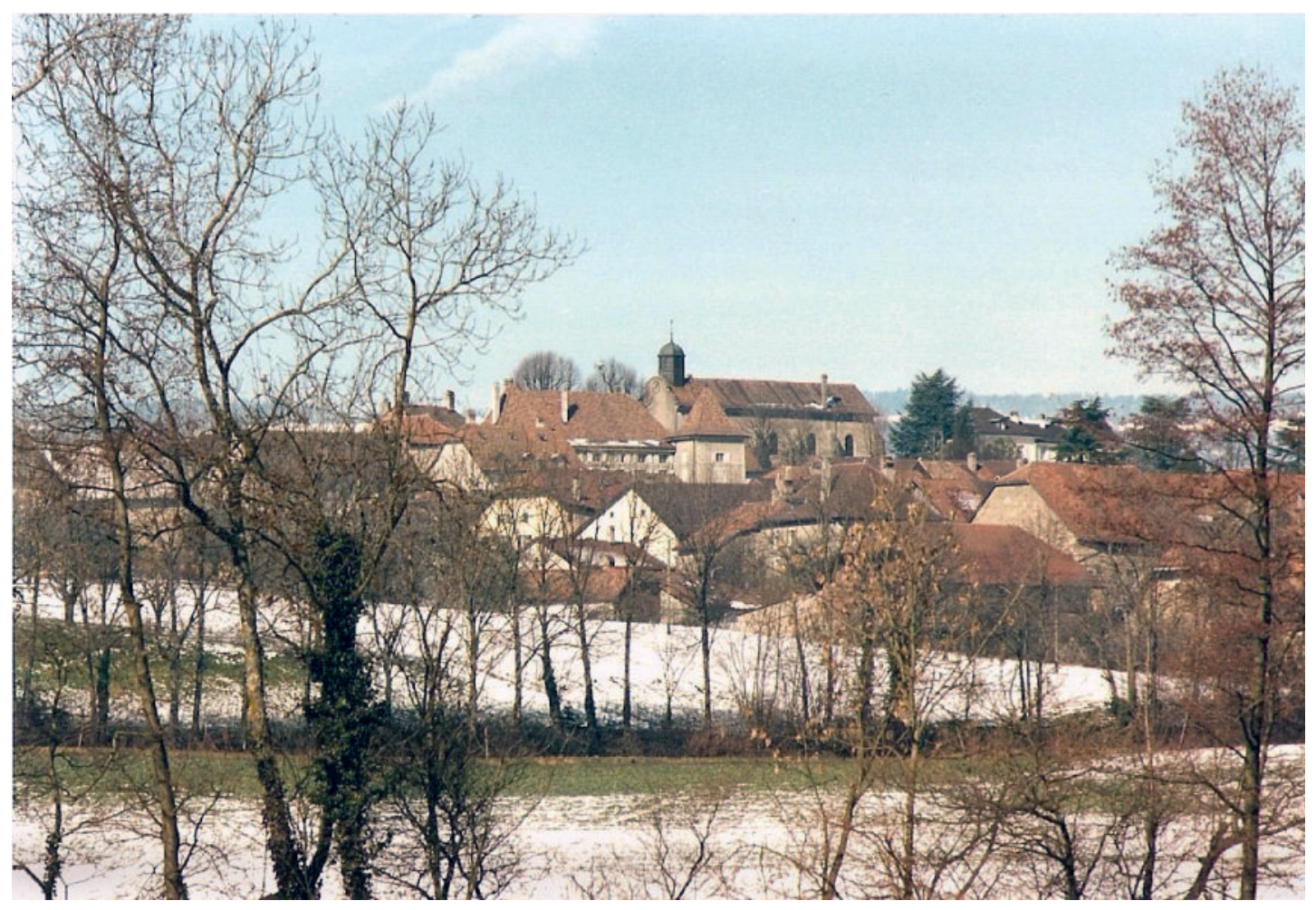


dépressions sont des situations fréquentes dans lesquelles le médecin généraliste, à la campagne comme en ville, a un rôle important à jouer, et où il peut «aider à vivre».

Les urgences dérangent, par définition pourraiton dire. Mais, comme le rappelait Sœur Julie, à l'Hôpital d'Aigle, «si on ne les accepte pas, on ne fait pas ce métier: on va mettre des cornichons en boîte dans la fabrique d'à côté». Cela dit, je me suis rendu compte au cours des années que, s'il est vrai que les urgences perturbent mon programme de consultations, elles

\section{Sur le plan professionnel, la campagne est l'endroit par excellence où le généraliste est à sa place}

gênent en général beaucoup moins les patients qu'il faut renvoyer de la salle d'attente: je les ai souvent entendu dire avec sagesse: «Une autre fois, ça pourrait être nous!»Et, lorsque le plâtre est fait, la suture terminée, la colique néphrétique maîtrisée, je me dis que l'imprévu a aussi ses bons côtés. Par ailleurs, comme chacun le sait, certains types d'urgence semblent avoir une prédilection pour les heures de la nuit; mais, là aussi, lorsque, après une demi-heure, on voit que le faux-croup est en train de passer, que l'œdème du poumon répond au traitement, que tout le monde se calme, on éprouve la satisfaction du travail réalisé sur place, avec des moyens simples, sans devoir recourir immédiatement à l'infrastructure hospitalière.

C'est un défi permanent que d'adapter ses connaissances à des situations réelles, c'est-à-dire là où les gens vivent et travaillent, et ce défi est stimulant pour qui aime faire preuve d'esprit pratique et d'indépendance. Les visites à domicile, qu'elles soient

\section{Rencontre}

La nuit est en train de tomber sur la campagne enneigée. Rentrant d'une dernière visite, je pense tout à coup à un patient qui a dû entrer aujourd'hui au CHUV pour une récidive de sa tumeur cérébrale. Je pense aussi à son père, qui normalement vit seul avec lui dans une ferme isolée, au pied des longues pentes boisées du Jura. Au cabinet, un dernier patient doit déjà être installé à la salle d'attente. Tant pis.

Mon arrivée devant la grande ferme est aussitôt signalée par le chien de garde. Inutile donc de frapper: j'approche de la porte d'entrée et j'attends un instant. Je vois bientôt arriver dans le corridor la silhouette chétive de celui qui a dû être autrefois un robuste paysan. Son visage inquiet s'illumine lorsqu'il me reconnaît. «Vous ne m'attendez pas!» «Eh bien non; entrez seulement!» Nous nous installons un moment à la grande table de la cuisine, sous le néon, près du fourneau potager dans lequel une nouvelle bûche vient d'être jetée. Le ronronnement du feu accompagne le tictac de la pendule. Un austère calendrier pend au mur blanc. La Bible est posée sur un rayon. Nous évoquons le temps où la table rassemblait plusieurs fois par jour toute la famille avec ses employés.

Quelques semaines plus tard, c'est le fils qui se rend à ma consultation. «ll paraît que vous êtes allé trouver mon papa! Ça lui a fait plaisir: il m'a dit: 〈ll y a un ange qui est venu!〉 Un ange, moi? Après tout..
}

en urgence ou de routine (il existe quelques personnes que je n'ai vues, pendant de nombreuses années, qu'à leur domicile), sont une occasion de sortir de ses murs et de se confronter à des réalités parfois insoupçonnées. On y fait souvent des observations conduisant à affiner son attitude médicale et à adapter les guidelines aux patients (et non pas l'inverse...). On prend conscience de ce que l'un de mes anciens professeurs appelait «ces petites choses qui font que la médecine marche ou qu'elle ne marche pas...» De plus, les patients apprécient ce genre de contact et ils nous en remercient parfois avec six œufs, une courge ou des noix. Et les trajets sont généralement pour moi un plaisir, l'occasion d'admirer le paysage, ou, en traversant les villages, de repenser à telle ou telle personne et de lui lancer une pensée au passage.

Lorsque «Infotrafic» m'annonce des embouteillages sur l'autoroute, et que je suis seul sur une belle route enneigée, je me sens bien là où je suis.

\section{Installation et débuts...}

C'est à Pampigny, village du pied du Jura vaudois situé à une quinzaine de kilomètres de Morges et une vingtaine de Lausanne, que ma femme et moi avons ouvert notre cabinet médical commun en 1978. A ce moment, la population de la région se composait essentiellement de gens travaillant la terre ou la forêt ou exerçant des activités artisanales dans les environs. Les villas étaient encore rares.

Loin de tout? Ce n'est pas mon sentiment. Comme bien des villages de la campagne, Pampigny se trouve à une distance raisonnable de la ville, et nous ne sommes pas isolés. Je peux affirmer que jamais, jamais, même en rentrant chez moi de nuit dans une tempête de neige après un concert, je ne me suis dit: «Mais qu'est-ce que je suis venu faire dans un coin pareil?» Pensons un instant à des endroits bien plus éloignés: il y a de nombreuses années, j'ai rencontré un médecin dans les îles Shetland, situées au nord de l'Ecosse. Seul pour les mille habitants de l'île d'Yell, il était passionné par son métier, mais regrettait tout de même de ne jamais pouvoir s'absenter une demi-heure la conscience tranquille. Et, bien sûr, les communications avec les autres îles étant ce qu'elles étaient à l'époque, ni lui ni ses patients n'avaient le choix. Pour ma part, j'apprécie de savoir que les patients qui viennent chez moi ne le font pas par obligation, mais parce qu'ils sont d'accord de travailler avec moi.

Voyons aussi tout ce qui est proche, et commençons par le cabinet médical, situé au rez-de-chaussée de la maison, ce qui évite la perte de temps quotidienne qui est par la force des choses le lot de tant de pendulaires. Il y a ensuite la campagne et la nature, littéralement à nos portes. Peu de moments sont aussi bienfaisants qu'une soirée de printemps où nous sortons à pied de la maison pour une balade dans la forêt. Le dimanche soir, une colonne de voitures 


\section{Rencontre}

Une colonne de fumée monte au-dessus de la forêt profondément enneigée. Quittant la route cantonale, je m'engage sur un chemin forestier pour aller saluer nos bûcherons. Ils sont sur le point d'abattre un grand sapin blanc. «ll doit tomber là, il faudrait pas qu'il aille «s'encrouer dans cette fourche de fayard!». Un dernier coup de tronçonneuse et, avec un craquement sec, le sapin tombe exactement à l'endroit désigné, entraînant un nuage de neige poudreuse. Les hommes procèdent ensuite à l'ébranchage, puis à l'écorçage au plumet. Tout à l'heure, ils se rendront dans la cabane forestière (le «refuge») pour leur repas de midi, pendant que je prendrai le mien bien au chaud avec ma famille.

redescend vers les villes. Nous, nous restons, nous serons là demain: c'est ici que nous avons notre lieu de vie et de travail.

Nous vivons parmi des communautés villageoises attachantes, avec lesquelles des liens solides se sont tissés au cours des ans. Depuis mon enfance, et les séjours à la montagne, j'ai toujours éprouvé une cer- à l'étable, matin et soir, les vaches doivent être traites. Il est vrai qu'avec eux, il faut parfois du temps pour aller au delà d'une certaine rudesse apparente et pour reconnaître ce qu'elle dissimule. Par ailleurs, une trentaine d'années en leur compagnie nous a fait vivre le passage des générations: ceux que je vois aujourd'hui sur les tracteurs sont en partie les anciens camarades d'école de mes enfants.

Souvent, les citadins imaginent et redoutent une certaine proximité avec la population, à laquelle ils préfèrent l'anonymat de la ville. Il nous arrive, c'est vrai, de devoir céder dans la rue au rite d'une petite conversation limitée à des banalités. L'autre côté de la médaille, c'est l'attention, en général discrète, que les gens se portent les uns aux autres, l'entraide, les services rendus en toute simplicité. Les rares fois où je me suis trouvé subitement sans voiture, je n'ai eu

\section{«Trente ans au même endroit, avec le même métier, cela peut paraître long, mais chaque jour apporte des choses nouvelles»}

taine fascination pour le monde paysan: des gens qui travaillent au rythme des saisons, avec des plantes et des bêtes. Comme nous, ce sont des indépendants, habitués à prendre des décisions et des risques dans leur profession. Ils ne vont pas s'aliter «pour la moindre des choses» - ils n'ont souvent pas le choix: aucune peine à m'en faire prêter une par un voisin.

Au cours des ans, nous avons développé tout un réseau d'amis dans la région (à lui seul, l'intérêt pour la nature constitue déjà un bon point d'accrochage et il crée des liens). De plus, il n'est pas difficile d'entretenir les contacts avec les amis vivant plus loin, qui

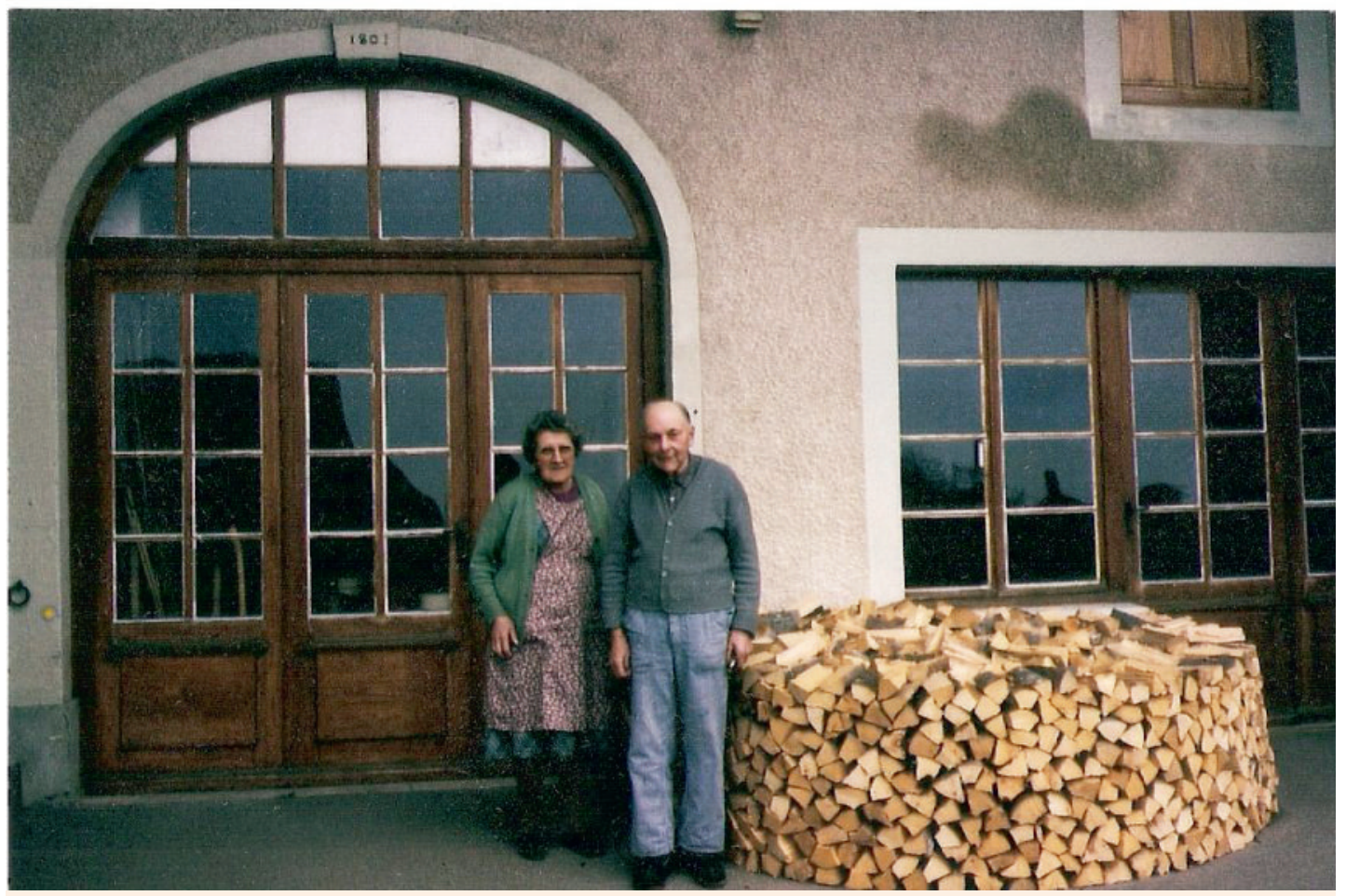

Il y a des patients que je n'ai vus, au cours des ans, qu'à leur domicile. 


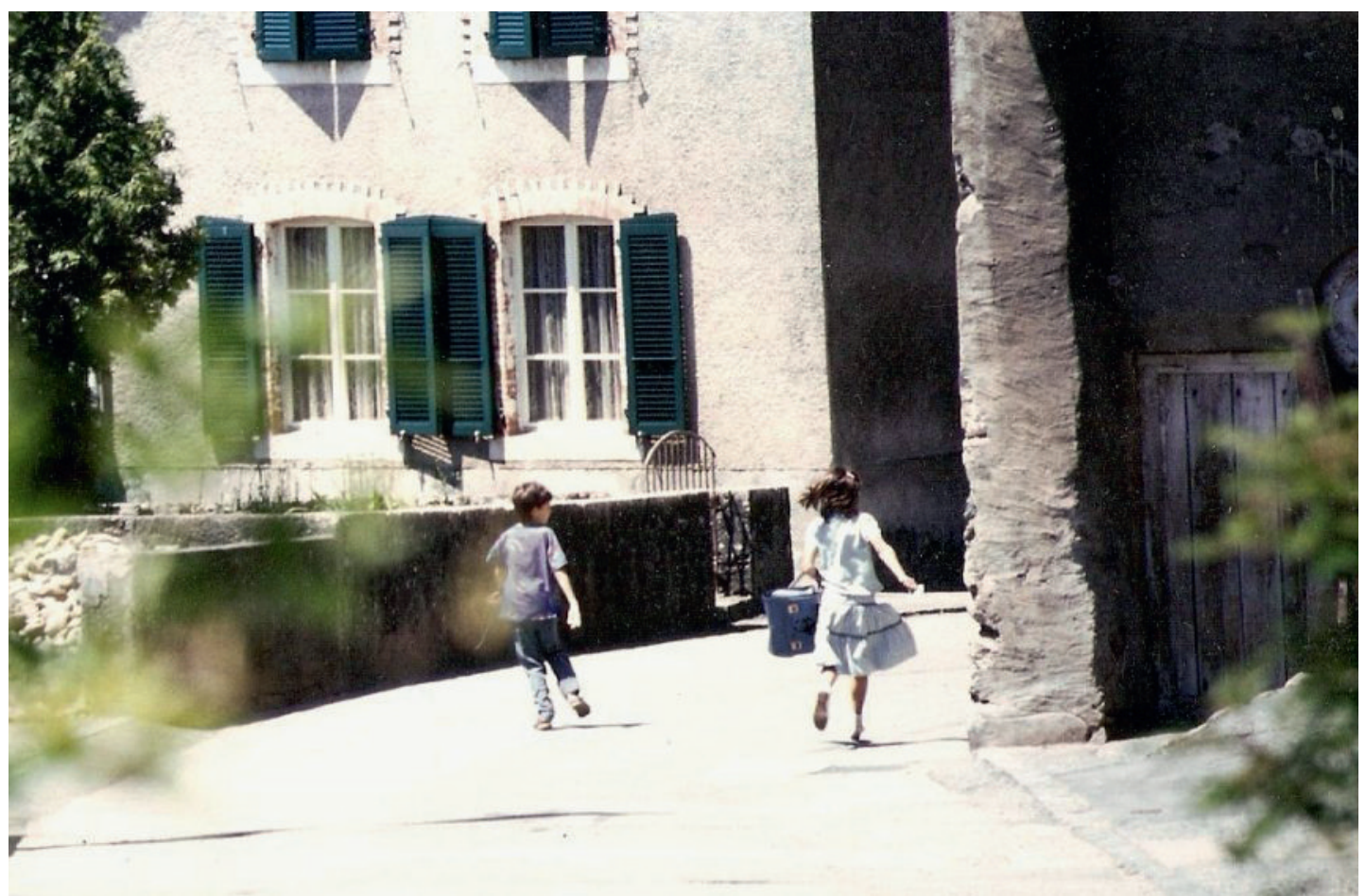

Nos enfants se rendent à l'école du village.

viennent toujours volontiers nous rendre visite à la campagne. Parmi eux, les citadins sont généralement frappés par un certain calme qui semble régner ici, qui se remarque encore plus nettement au cours de la soirée ou de la nuit. A ces moments, aux quelques bruits de la circulation succèdent la cloche de l'église sonnant les heures et celles des vaches qui paissent, le ronron d'un avion lointain, peut-être le souffle du vent dans la ramure des arbres. Le paysage avec ses étendues, l'espace qui nous entoure y jouent sans doute un rôle important. On se souvient en effet de l'expérience réalisée par des éthologues qui avaient introduit de petits poissons dans un aquarium se rétrécissant progressivement: au fur et à mesure que la distance disponible entre les individus diminuait, les poissons devenaient plus agités et agressifs. Belle démonstration de l'importance de disposer d'un espace vital suffisant. Ce calme constitue aussi une ambiance appréciable pour travailler, même s'il ne doit pas faire illusion quant à la réalité: laissons les poètes à leurs

\section{Rencontre}

Au retour d'une visite une nuit de printemps, sortant de la forêt, je m'arrête, j'éteins les phares et je m'éloigne de la voiture. Les prés sont inondés de lumière par la pleine lune: on pourrait presque voir passer un chevreuil le long de la lisière. C'est un moment exceptionnel qui mérite que je renonce à un peu de sommeil. Bientôt, un son mélodieux vient du ciel, d'abord faible, puis s'amplifiant: les voix flûtées, mélancoliques, merveilleuses, d'un vol de courlis en migration! Se dirigeant par les étoiles, les invisibles échassiers volent vers le nord, et je rêve un moment aux landes et aux tourbières d'Ecosse, au vent de l'Atlantique qui met du sel sur les lèvres, aux nuages gris promettant une prochaine averse... rêveries bucoliques, il n'y a pas de raison que les drames, la souffrance, la violence épargnent la campagne.

Le généraliste de campagne connaît bien ses collègues des villages voisins avec lesquels il est habitué à collaborer, de même que chacun des spécialistes de la ville la plus proche auxquels il réfère ses patients pour avis. Il connaît aussi les hôpitaux voisins, dans mon cas ceux de Morges et de Saint-Loup, au sein desquels il entretient des contacts personnels qui sont

\section{«Tout est donc parfait? Mais non,}

\section{bien sûr!»}

bien préférables à l'anonymat des grands ensembles. Il est en outre l'interlocuteur des infirmières et auxiliaires des centres médico-sociaux dont le travail est tant apprécié. Il peut se voir appelé à soigner une personne en fin de vie, désirant passer ses derniers jours parmi les siens. Une telle demande implique souvent un investissement conséquent et une grande disponibilité à l'égard du patient, de sa famille et de tous les autres intervenants, mais ce sont là des moments forts pour lesquels l'entourage sera souvent reconnaissant. Lorsque, un beau jour, dans de lointains bureaux, on a inventé le concept de «réseau», nous avons été nombreux à sourire et à nous dire que cette nouveauté, c'était ce que nous pratiquions tout natu- 
rellement depuis toujours, et avant nous nos prédécesseurs, sans toutefois que personne n'ait jamais ressenti la nécessité d'y mettre un nom.

\section{La famille}

Nos enfants ont aimé grandir à la campagne, aller à l'école du village, puis descendre en ville pour le niveau secondaire. Même s'il leur fallait se lever tôt pour prendre le train de sept heures, les trajets en transports publics leur ont surtout laissé le souvenir des bons moments passés avec leurs camarades des nombreux villages voisins. Ils ont tous les deux fait des études universitaires et vivent actuellement à Lausanne.

Pour les enfants comme pour leurs parents, la vie à la campagne est riche en occasions de rencontre et de loisirs: terrain de football, salle de gymnastique, manèges, diverses possibilités d'activités artistiques, notamment musicales, paroisse, sociétés de jeunesse, de chant et bien d'autres. Souvent, des groupes, parfois informels, organisent une soirée, une exposition ou une rencontre sur tel ou tel thème. A deux minutes de chez nous se trouvent la poste, la boulangerie, le magasin d'alimentation, la petite banque Raiffeisen. «Mon seul regret», me disait récemment une jeune mère de famille ayant quitté Genève deux ans auparavant, «c'est de ne pas l'avoir fait plus tôt».

\section{Et si c'était à refaire?}

Si vous m'avez lu jusque là, vous aurez deviné ma réponse. Trente ans au même endroit, avec le même métier, cela peut paraître long, mais chaque jour apporte des choses nouvelles, les gens changent, la médecine évolue. Bien des certitudes médicales ont fait place à d'autres certitudes.

Tout est donc parfait? Mais non, bien sûr! Je connais aussi des gens pénibles, des quérulents, des malhonnêtes et même quelques mauvais payeurs! En réalité, ils ne sont qu'une infime minorité, et, de plus, les années de participation à un Groupe Balint m'ont aidé non seulement à les accepter, mais aussi à mieux les comprendre, et donc à mieux décoder leur demande. Je me souviens aussi, et cela me touche plus, de ces cas de cancer que j'aurais voulu avoir décelés plus tôt, ou d'autres situations où j'aurais dû être meilleur. Sans doute le malaise ressenti peut-il être plus profond à la campagne que dans le relatif anonymat de la ville. Un confrère lausannois, diabétologue expérimenté, confiait un jour à notre groupe de généralistes: «Si vous faites une erreur grave à Pampigny, vous y êtes grillé; si c'est moi, il me reste toujours le reste du canton!» J'ai admiré sa sincérité tout autant que son réalisme. Enfin, et c'est normal, il y a des jours où l'on a tout naturellement un peu moins envie de travailler que d'autres.

Cela dit, je suis bien conscient des difficultés administratives et économiques menaçant l'activité médicale, et tout particulièrement celle des généralistes. Une grande partie de la responsabilité en incombe à l'ancien ministre de la santé, Pascal Couchepin, qui ne s'intéressait guère à la santé, ni à la médecine, si ce n'est en termes purement économiques. Pour faire bon compte, il lâchait occasionnellement de petites phrases louant le rôle-clé des généralistes dans le système de santé du pays, mais en même temps il semblait faire tout ce qu'il pouvait - et il pouvait beaucoup - pour les décourager. J'ai écrit un jour dans la presse que nous n'avions pas besoin que Pascal Couchepin nous aime, seulement qu'il nous laisse travailler.

Il faut souhaiter, pour les médecins et avant tout pour les patients, que son successeur Didier Burkhalter fasse preuve d'une approche plus large, plus humaniste, plus proche de la réalité et de la vie des gens, et que l'administration évolue elle aussi dans ce sens. Nos souhaits ne suffiront toutefois pas et sans doute nos successeurs devront-ils aussi lutter, faire connaître le sens de leur activité et présenter une image de la médecine un peu plus attrayante qu'une page excel avec des chiffres.

Ma femme a cessé son activité professionnelle il y a bientôt deux ans. Par la suite, une jeune collègue interniste, amie de notre fille, est venue s'installer au cabinet à un taux de $40 \%$. Nous avons conclu un contrat d'association, pour lequel nous avons bénéficié des conseils de la Société Vaudoise de Médecine ainsi que de la part de quelques collègues ayant passé par un cheminement analogue. L'accueil que lui ont réservé les patients montre, si c'était nécessaire, à quel point la médecine générale répond toujours à un besoin. Un jour, j'espère, une autre personne viendra la rejoindre, et je me retirerai en laissant aller cette jeune équipe.

C'est une fin d'après-midi d'été. Quelques cumulus vagabondent au-dessus de la longue silhouette du Jura. Des enfants jouent dans la rue; trois moineaux piaillent au bord d'un toit. Dans le village flotte l'odeur du foin fraîchement récolté. Je descends à la laiterie pour y faire quelques achats habituels. Quand ils étaient petits, l'un ou l'autre de mes enfants m'y accompagnait souvent.

Voilà une manière bien agréable de prendre l'air après une journée de travail. Celle de ce lundi était assez animée, et elle s'est bien passée. J'échange quelques mots avec les paysans venant livrer le lait de la traite du soir. Eux aussi sont au terme d'une journée de travail au village. Un jour, ils auront dû faire face à des incidents ou des difficultés inattendues; une autre fois, ils auront eu la satisfaction d'avoir pu rentrer la moisson juste avant l'orage...

Un paysan âgé, ayant remis le domaine à son fils, m'a dit un jour: «Paysan, c'est le plus beau métier qui soit!» Je me suis dit alors à moi-même: "Médecin de campagne aussi!». 